\title{
Pharmacotherapy and child psychiatry: is there a way forward?
}

\author{
INVITED COMMENTARY ON... CHILD PSYCHIATRY AND ITS RELATIONSHIP \\ WITH THE PHARMACEUTICAL INDUSTRY ${ }^{\dagger}$
}

A. James

Abstract Recent controversy over the use of serotonin reuptake inhibitors in children and adolescents has
focused attention on the role of the pharmaceutical industry in the treatment of young people. Failure
of pharmaceutical companies to fully disclose negative outcome trials has led to new guidelines
for publication of all trial results. Scrutiny is on the conduct of trials and the relationship of the
pharmaceutical industry with prescribing doctors and post-trial surveillance of new drugs. It is argued
that drug treatments in child psychiatry are a powerful therapeutic tool but vigilance is needed to ensure
that data on the efficacy and safety of drugs are freely available.

There has been considerable controversy over the role of pharmacological treatments for psychological disorders in children and adolescents. Revelations by the media about the lack of efficacy and harmful effects of selective serotonin reuptake inhibitor antidepressants (SSRIs), and the practice within parts of the pharmaceutical industry of hiding or failing to disclose negative drug trials, have heightened this controversy. The fact that these problems were made public by a few individual doctors and journalists rather than academic establishments or learned bodies is a further cause for concern.

The relationship between psychiatry and the pharmaceutical industry has not always been straightforward. Indeed, the discovery of many of the major drug treatments such as antipsychotics and antidepressants initially owed more to serendipity than to a theoretically informed development. However, there can be little argument that first-generation antipsychotic medications have benefited millions of patients since their introduction in the 1950s. Likewise, clinical practice and numerous double-blind randomised controlled trials (RCTs) have shown the benefits, at least in adults, of antidepressant medication, although recent evidence does question the side-effect profile and efficacy of SSRIs in younger adults (Friedman \& Leon, 2007).

${ }^{+}$See pp. 3-9, this issue.
There has been a huge increase in the rate of prescribing of medication for psychiatric disorders in children and adolescents. While this, in part, may be due to an increased recognition of disorders and demand for treatment, there is some cause for concern. For instance, in the USA there was a six-fold rise in the prescribing of antipsychotic medications between 1993 and 2002 (Olfson et al, 2006) and over one-third of these prescriptions are for children with mood disorders, an indication for which the evidencebase is lacking. Alongside this there is a trend for increased prescribing ahead of evidence. For example, although only a few RCTs show risperidone to be of benefit to children with disruptive behavioural disorders and a diagnosis of autism or pervasive developmental disorder (McCraken et al, 2002; Shea et al, 2004; Padina et al, 2006), there is widespread use of antipsychotics for the treatment of disruptive behavioural disorders in children in general (Olfson et al, 2006). Recently, the licence holder (JanssenCilag) applied to the UK licensing authority, the Medicines and Healthcare products Regulatory Agency (MHRA), to include irritability in autism as a licensed indication. After seeking views of experts and the National Autistic Society, the Committee on Safety of Medicines offered conditional approval, dependent on safety monitoring with written reports to MHRA. Unfortunately, the company withdrew its application (Morgan \& Taylor, 2007). Thus, despite agreement on the use of risperidone for the

Anthony James is a consultant child and adolescent psychiatrist at Highfield Adolescent Unit (Warneford Hospital, Oxford OX3 7JX, UK. Email: tony.james@obmh.nhs.uk) and Honorary Senior Lecturer at the University of Oxford. His research interests include neuroimaging of early-onset psychosis, obsessive-compulsive disorder and eating disorders. 
management of aggression, a valuable opportunity for safety monitoring was lost and no doubt the medication will continue to be prescribed unlicensed. There is also a trend for use of this antipsychotic in children with a diagnosis of conduct disorder. Indeed, what evidence there is suggests that the effect size for a reduction of aggression in the case of attention-deficit hyperactivity disorder (ADHD) and conduct disorder is very modest (Armenteros et al, 2007). There is a concern that the widespread use of antipsychotics in youngsters might cause adverse neurological side-effects such as tardive dyskinesia, although a recent review (Padina et al, 2006) showed that the rate of side-effects with lowdose antipsychotics was low.

Another area of concern, highlighted by the US Institute of Medicine of the National Academies (2006), centres on the system for ensuring the safety of drugs after their approval by the Food and Drug Administration (FDA). It has been argued (Hennessy \& Strom, 2007) that limitations in the pre-approval drug-testing process have resulted in $20 \%$ of drugs receiving black box warnings after approval, and $4 \%$ of drugs being ultimately withdrawn from the market for safety reasons. Curiously, in a wealthgenerating industry with substantial applicant fees, financial limitations at the heart of the FDA organisation have been identified as one possible reason for shortcomings.

\section{The problem of bias}

Belief in the scientific underpinnings of psychopharmacology is essential, and representation of research findings is an important part of that process. Any corruption of this information is absolutely to be decried. In relation to drug trials, there are two main sources of bias: first, biases in trial design and, second, biases in dissemination of results (Vieta, 2007). Vieta's concerns focus on industry-funded drug trials for adults, but the principles are pertinent to all ages. Even though the FDA in North America and the European Medicines Agency (EMEA) (formerly the European Agency for the Evaluation of Medicinal Products) have set design guidelines for drug trials, trial design biases are still possible (Box 1). According to Vieta (2007), biases in dissemination of results (Box 2) can be more difficult to detect, but it is clear that publishing negative trials is the responsibility of all those involved in the research process, including journal editors. There is an added ethical issue: patients who voluntarily participate in trials with the expectation that their input will contribute to furtherance of scientific knowledge and be placed in the public domain have rights that need to be respected ahead of commercial interests.

\section{Box 1 Potential biases in drug trial design}

- False non-inferiority designs

- Enriched designs

- Underpowered comparator samples

- Unfair comparator doses

- Inclusion of patients who are nonresponsive to the comparator

- Unfair rescue medication rules

- 'Creative' outcome measures favouring the drug of choice

(Vieta, 2007)

No one can support the failure to disclose negative trial results. Furthermore, Timimi's criticisms of the conduct of a number of the trials involving fluoxetine are legitimate, in that primary outcome measures were often not significant and secondary outcome measures were reported as being significant (Timimi, 2007, this issue). This is clearly wrong and against the recommendations set out in the latest guidance on design and analysis of clinical trials (Food and Drug Administration, 1998), which state that primary outcome variables in regulatory trials must 'provide a valid and reliable measure of some clinical and relevant and important treatment benefits in a patient population'.

Pharmaceutical industries are multibillion-pound, worldwide enterprises; it is possible that financial pressures underlie some of the malpractice in reporting drug trial results. As part of regulation transparency and policies of disclosure are the barest minimum standards that must be set. Cynically one might believe that large multinational companies are unlikely to respond solely to regulatory bodies, academics or editors of journals, often citing commercial sensitivities as a reason for not disclosing information. Is it likely, therefore, that what is required, as has been shown with the tobacco

\section{Box 2 Potential biases in dissemination of} results

- Suppression of the publication of negative results, which remain 'data on file', while positive results are published

- Presentation of negative results as posters at conferences, on small websites or in a very conservative format

- Analysis, sub-analysis and repeated publication of positive results, and presentation at scientific meetings

(Vieta, 2007) 
industry, is group legal action in US Courts taken by individuals - patients experiencing drug sideeffects or relatives of patients who took their own lives. The likelihood of financial penalties is often a major driver for change in practice.

\section{The persuaders?}

The ubiquitous nature of pharmaceutical company sponsorship in the USA and probably the UK is quite astounding (Campbell et al, 2007) - apparently around $93 \%$ of doctors accept gifts, drug samples or conference expenses. Timimi and others accuse medical healthcare professionals of, at best, naïvety. Do doctors change their prescribing practices in response to pharmaceutical company promotions? According to Timimi we do. However, the major changes in my prescribing practice - use of secondgeneration antipsychotics and long-acting stimulant formulations - were made to reduce side-effects and for ease of use and adherence respectively, rather than in blind response to pharmaceutical company promotions.

The claim that psychiatrists are unduly influenced by pharmaceutical company promotions can be examined by more than anecdotal evidence. In the case of eating disorders - bulimia nervosa in particular - guidelines from the UK's National Institute for Health and Clinical Excellence (NICE) recommend cognitive-behavioural therapy (CBT) as first-line treatment, with antidepressant medication as an alternative or addition as necessary (National Collaborating Centre for Mental Health, 2004). Whether or not child psychiatrists follow these guidelines is more likely to depend on the availability of resources to deliver CBT. If, however, CBT is available then in my experience child psychiatrists do use psychological treatments first. A recent evidence-based manual for child psychiatry (Wolpert et al, 2006) recommended medication as the first-line treatment for only three out of fourteen disorders - ADHD, psychosis and Tourette syndrome. This suggests that child psychiatry is, if anything, psychotherapeutically oriented rather than drug dominated.

\section{The profit incentive}

Given the recent disclosures (e.g. Healy et al, 1999; Panorama, 2007), Timimi's view of pharmaceutical companies driven by commercial rather than scientific concerns may be difficult to refute. Tight regulatory mechanisms are needed. However, it is surely not in the interests of pharmaceutical companies to promote ineffective or harmful drug treatments. Indeed, there is accumulating evidence from meta-analyses showing moderate benefits for drug treatments in child psychiatry in the areas of depression, obsessive-compulsive disorder and anxiety disorders, and large effects for ADHD (see below).

Although it is clear that one of the prime aims of pharmaceutical companies is to make profits for their shareholders, the spin-offs are not inconsiderable. For example, one pharmaceutical company has recently set up a UK centre for translational medicine, with huge resources devoted to neuroimaging. The ultimate aim is the development of drug treatments, but it is clear that the concomitant developments in neuroimaging and neuroimaging techniques are likely to lead to considerable insights that will ultimately benefit patients. It has been argued that the pharmaceutical industry invests $£ 3.3$ billion in research and development in the UK each year, and in 2003 it contributed a positive trade surplus of $£ 3.6$ billion. According to the Association of the British Pharmaceutical Industry (2007), over 300000 jobs rely on the industry. Furthermore, pharmaceutical companies contribute significantly to nonpharmaceutical research projects (Goodwin, 2007).

\section{Pharmaceutical company sponsorship}

Worryingly, pharmaceutical company promotions are not limited to doctors - there has also been a growth in the funding of patient groups (Kent \& Mintes, 2007). Is it a concern, for instance, that pressure or lobby groups funded by pharmaceutical companies challenge the NICE guidelines over the use of cholinesterase inhibitors in Alzheimer's disease, despite the apparent lack of demonstrable evidence for their efficacy in certain stages of the illness?

\section{Off-label prescription}

Until recently, a major problem for child psychiatry has been that a large proportion of the medications we use have been unlicensed. In January 2007 the Paediatric Medicine Regulation came into force. Pharmaceutical companies will be expected to produce a 'paediatric investigation plan' (PIP) for all new medicines. These will be submitted to the EMEA's Paediatric Committee, which will be responsible for determining whether the medicine is likely to be of potential therapeutic benefit to children. This will apply to all new drugs unless they are for conditions occurring only in adulthood, or likely to be ineffective or unsafe in children. All results of 
PIP trials will be held on a EU database. Medicines already in use will need a paediatric marketing authorisation. Companies will be given a financial incentive to carry out the necessary extra studies via a 6-month extension to their supplementary patent protection. This generous inducement should result in more clinical trials involving paediatric patients. Alongside regulatory changes in the USA there has been an upsurge of interest in the practice of paediatric psychopharmacology (DeVeaugh-Geiss et al, 2006). Other US initiatives have included the setting up of an infrastructure for paediatric psychopharmacology research (the Child and Adolescent Psychiatry Trials Network, CAPTN) (Research Unit on Pediatric Psychopharmacology Anxiety Study Group, 2001; March et al, 2004a), as well as government-funded comparative treatment trials of medications and psychotherapy (MTA Cooperative Group, 1999; March et al, 2004b; Pediatric OCD Treatment Study Team, 2004).

\section{Safety and efficacy}

One of the major concerns for psychopharmacology research is the detection of rarer safety issues, not always detectable by traditional clinical trials and statistical approaches. To establish a statistical link between a treatment and rare events requires impractical sample sizes; alternatives such as longterm follow-up and statistical methods for evaluating selected groups have been suggested (DeVeaughGeiss et al, 2006). DeVeaugh-Geiss et al argue that in the USA, tight bureaucratic procedures affect drug trials: frequently, acute-dose pharmacokinetic studies have not been completed before initiation of efficacy and safety studies; efficacy and safety trials may be conducted at doses that are inappropriate for demonstrating efficacy or tolerability in children and in adolescents; and population pharmacokinetic trials remain rare.

Previously research and trial studies have been limited typically to efficacy studies, mostly using the gold standard approach of RCTs. Unfortunately, these trials frequently exclude most comorbidity, and are often short-term and too small to identify subgrouping variables or other than common adverse events. Ethical and other considerations have spurred researchers to look at alternatives to the classical RCT (March et al, 2004c). Future studies are likely to include: active controlled noninferiority (or superiority) trials, adaptive designs, group sequential designs, patient preference designs and equipoise-stratified designs (DeVeaugh-Geiss et al, 2006). Nevertheless, it is clear that what is also needed is effectiveness trials. The establishment of large, simple effectiveness trials conducted via practical clinical trials networks is therefore a major step forward (March et al, 2005). With sufficient power, large multicentred trials will allow not only the setting up of safety databases to define the risks and tolerability of drugs in the short and long term, but also identification of moderators of treatment outcome.

Another innovation in psychopharmacology research, perhaps belatedly, has been the inclusion of psychotherapy during trials (March et al, 2004b; Goodyer et al, 2007). One could argue that, with renewed academic input, regulatory changes and effective trial forums, more reliable psychopharmacology data should be become readily available.

\section{Pharmacotherapy of ADHD}

Timimi is correct in pointing to some of the deficiencies in the Multimodal Treatment Study of Children with ADHD (MTA) (MTA Co-operative Group, 2004), which have been incorrectly interpreted by some as a solely positive endorsement for the superiority of drug treatments. However, the treatment of ADHD is one of the most researched areas in medicine, with meta-analyses showing that pharmacological treatment has a substantial effect on the core features of the disorder (attention, impulsivity, hyperactivity) (Crenshaw et al, 1999; Faraone \& Biederman, 2002). The benefits of long-term surveillance is demonstrated by the finding that, although the MTA study showed an advantage of stimulant medication and combination treatments at 14 and 24 months, the 3-year follow-up (Jensen et al, 2007a) showed equal benefits for all four treatments - stimulant medication, behavioural therapy, a combination of both, and community care as usual. Interestingly, there was a significant reduction in those taking medication during the follow-up period (a drop from $91 \%$ to $71 \%$ ), and an increase in those undertaking behavioural therapy (from $14 \%$ to $45 \%$ ). A recent meta-analysis (Majewicz-Hefley \& Carlson, 2007) of eight studies has shown large effect sizes for combined treatments, the psychosocial treatments having differing effects on the peripheral features of the disorder (deficits in social skills, academic performance and following directions). The combined treatment approach confirms the recommendations of the American Academy of Child and Adolescent Psychiatry (1997).

\section{Pharmacotherapy of depression}

There has been extensive debate about the safety of antidepressants for the treatment of anxiety and 
depression in children and adolescents. In June 2003 the UK's MHRA and the US FDA advised that paroxetine should not be prescribed for anyone under 18 years of age. After a re-evaluation of data on 4582 paediatric participants in 24 antidepressant trials of 4-16 weeks' duration (23 industry-sponsored trials and the Treatment of Adolescent Depression Study) (Hammad et al, 2006) it was concluded that between $1 \%$ and $3 \%$ of treated patients could be at risk of antidepressant-induced suicidality, although no suicides had occurred.

One of the major methodological shortfalls in the meta-analyses of the relationship between suicide and SSRIs has been the classification of suicidal events, which is haphazard and therefore unreliable. Even with a reliable classification system for suicidal events (Posner et al, 2007), it is clear that only spontaneously reported adverse events are available for classification. Furthermore, the effect of medication on the threshold for spontaneous reporting between treatments and on actual suicidal ideation and acts remains unclear. Interestingly, however, an increase in the risk for spontaneously reported suicidal events in those taking antidepressant medication was associated with a tendency towards a protective effect against new-onset and worsening suicidal ideation (Hammad et al, 2006).

In the USA, a black box warning on the labelling of all antidepressants for use with children and adolescents and a patient medication guide was mandated in 2005. The immediate effect (within 24 months) of the FDA black box warning was a significant reduction in diagnoses of paediatric depression and prescribing of SSRIs to children and adolescents by primary care physicians (Libby et al, 2007). Worryingly, following the FDA black box warning there has been an $18 \%$ rise in the US adolescent suicide rate (Hamilton et al, 2007). These findings need to be set in the context of the indirect evidence of an association between the increased prescription of SSRIs and a reduced youth suicide rate (Olfson et al, 2003; Gibbons et al, 2006), indicating a protective effect of SSRIs against suicide.

The largest and most recent meta-analysis of 27 RCTs of antidepressant treatments involving 5310 children and adolescents (Bridge et al, 2007) looked at major depressive disorder, anxiety disorder and obsessive-compulsive disorder. According to the study there was a small response rate (pooled risk differences) for major depressive disorder $(11.0 \%$; 95\% CI 7.1-14.9), and a larger response for obsessivecompulsive disorder (19.8\%; 95\% CI 13.0-26.6), with the largest response for anxiety disorder $(37.1 \%$; 95\% CI 22.5-51.7). The corresponding numbers needed to treat were 10 (95\% CI 7-15), 6 (95\% CI $4-8)$, and 3 (95\% CI 2-5) respectively. There was increased risk difference of suicidal ideation/suicide attempt across all trials for drug $v$. placebo $(0.7 \%$; $95 \%$ CI $0.1-1.3)$, with a number needed to harm of 143 (95\% CI 77-1000). Importantly, there were no completed suicides and, as has been pointed out, the rate of suicidal ideation in the trials is low and comparable to that seen in a normal adolescent population (James, 2005). Age-stratified analyses showed that for children under 12 years old with major depressive disorder, only fluoxetine showed benefit over placebo, which accords with the current prescribing guidelines.

An important question is whether combination treatments are more effective. The NICE guidelines for the treatment of depression in children and adolescents (National Collaborating Centre for Mental Health, 2005), which Timimi does not mention, recommend psychological treatments as first line for mild and moderate depression. However, although the Treatment of Adolescent Depression Study showed the benefits of combined CBT and fluoxetine (March et al, 2006), a more recent study from Cambridge and Manchester, which was more representative of routine clinical practice in child and adolescent mental health services (Goodyer et $a l, 2007)$ demonstrated that in moderate and severe depression pharmacological treatment with an SSRI alone is effective, and CBT confers no additional benefit. This accords with the view expressed by Goodwin (2007) that drug-placebo differences seem to be magnified with illness severity, whereas psychotherapy tends to look less effective as illness severity increases.

\section{Conclusions}

It is clear that restoration of public and medical confidence in the pharmaceutical industry will require changes in practice. There is agreement that, at the very least, disclosure of researchers' interests and all trial outcomes are necessary. A few further suggestions could be made.

First, in the UK a strong case could be made for the setting up of academic departments of child psychopharmacology. These would be involved in conducting drug trials and disseminating findings. Such departments could be partly funded through NHS research and development schemes and partly by the pharmaceutical industry. The latter funding would need to be open and subject to scrutiny. Already there is a Centre for Interventional Paediatric Psychopharmacology at Great Ormond Street Hospital for Children in London.

Second, the International Committee of Medical Journal Editors (ICMJE) initiated a policy in 2005 requiring investigators to deposit information about trial design into an accepted clinical trials 
registry before any patient involvement (Laine $e t$ $a l, 2007)$. Despite initial hesitation, the story is of considerable success. For example 1 month after this policy came into effect, registrations with ClinicalTrials.gov (run by the US National Institutes of Health) had increased from 13153 to 22714 , and the number now stands at over 40000 . The World Health Organization is supporting an International Clinical Trials Registry Platform (ICTRP), which will include preliminary pre-trial pharmacokinetic studies. As regards publication, the ICMJE favours a standard abstract-reporting process, which is likely to be supported in forthcoming guidelines from the Consolidated Standards of Reporting Trials (CONSORT) group (Keech et al, 2007). Prior to publication full disclosure of all related postings in registries will be required. Hopefully this will lead to a reduction in misinformation and, as a recent $B M J$ editorial (Laine et al, 2007) states, 'will [help] to assure trial participants that the information that accrues as a result of their altruism will become part of the public record'.

Third, it is clear that no matter how rigorous clinical trials are, the number of participants involved is relatively small. Post-trial drug surveillance is dependent on practitioners reporting adverse effects using systems such as the UK's Yellow Card Scheme. Perhaps we are all guilty of underreporting, otherwise how could the SSRI story have gone undetected for so long? There is a welcomed proposal, supported by the Mental Health Research Network, to set up a national child and adolescent psychiatric surveillance system in the UK.

One hopes that the ongoing debate regarding drug treatments will have beneficial effects. One example is the publication of evidence-based treatment algorithms (Hughes et al, 2007). Nevertheless, caution needs to be exercised when extending drug treatments outside diagnostic groupings, as for instance in the management of impulsive aggression. It is welcome, then, that a recent academic review highlighted this particular issue and offered advice to guide future research strategies (Jensen et al, 2007b).

It would be naïve and probably wrong to say that all research misinformation is confined to drug trials. Side-effects and problems with treatment are not confined to drugs and occur in psychological treatments as well, but these are rarely, if ever, reported. Undoubtedly they should be.

Timimi's article has highlighted a number of extremely important issues. One welcomes the opportunity to critically examine the role of psychopharmacology in child and adolescent psychiatry. It is evident that some progress has been made in addressing very legitimate concerns; however, there is still some way to go.

\section{Declaration of interest}

A. J. has worked as a consultant to Sanofi Aventis and works collaboratively on a neuroimaging project with researchers from GlaxoSmithKline.

\section{References}

American Academy of Child and Adolescent Psychiatry (1997) Practice parameters for the assessment and treatment of children, adolescents, and adults with attention-deficit/ hyperactivity disorder. Journal of the American Academy Child and Adolescent Psychiatry, 36 (suppl. 10), 85S-112S.

Armenteros, J., Lewis, J. \& Davalos, M. (2007) Risperidone augmentation for treatment-resistant aggression in attentiondeficit/hyperactivity disorder: a placebo-controlled pilot study. Journal of the American Academy Child and Adolescent Psychiatry, 46, 558-565.

Association of the British Pharmaceutical Industry (2007) 'Information \& statistics'. ABPI. http://www.abpi.org.uk/ information/default.asp

Bridge, J., Iyengar, S., Salary, C., et al (2007) Clinical response and risk for reported suicidal ideation and suicide attempts in pediatric antidepressant treatment: a meta-analysis of randomized controlled trials. JAMA, 297, 1683-1696.

Campbell, E., Gruen, R., Mountford, J., et al (2007) A national survey of physician-industry relationships. New England Journal of Medicine, 356, 1742-1750.

Crenshaw, T., Kavale, K., Forness, S., et al (1999) Attention deficit hyperactivity disorder and the efficacy of stimulant medication: a meta-analysis. Advances in Learning and Behavioral Disabilities, 13, 135-165.

DeVeaugh-Geiss, J., March, J., Shapiro, M., et al (2006) Child and Adolescent Psychopharmacology New Millennium: a workshop for academia, industry, and government. Journal of the American Academy Child and Adolescent Psychiatry, 45, 261-270.

Faraone, S. \& Biederman, J. (2002) Efficacy of Adderall ${ }^{\circledR}$ for attention-deficit/hyperactivity disorder: a meta-analysis. Journal of Attention Disorders, 6, 69-75.

Food and Drug Administration (1998) International Conference on Harmonisation: guidance on data elements for transmission of individual case safety reports. Federal Register, 63, 2396-2404.

Friedman, R. \& Leon, A. (2007) Expanding the Black Box Depression, Antidepressants, and the Risk of Suicide. New England Journal of Medicine, 356, 2343-2346.

Gibbons, R., Hur, K., Bhaumik, D., et al (2006) The relationship between antidepressant prescription rates and rate of early adolescent suicide. American Journal of Psychiatry, 163, 1898-1904.

Goodwin, G. (2007) Conflicting interests and doing right. World Psychiatry, 6, 25-26.

Goodyer, I., Dubicka, B., Wilkinson, P., et al (2007) Selective serotonin reuptake inhibitors (SSRIs) and routine specialist care with and without cognitive behaviour therapy in adolescents with major depression: randomised controlled trial. BMJ, 335, 142. doi: $10.1136 /$ bmj.39224.494340.55

Hamilton, B., Minino, A., Martin, J., et al (2007) Annual summary of vital statistics: 2005. Pediatrics, 119, 345-360.

Hammad, T., Laughren, T., Racoosin, J. (2006) Suicidality in pediatric patients treated with antidepressant drugs. Archives of General Psychiatry, 63, 332-339.

Healy, D., Langmaak, C. \& Savage, M. (1999) Suicide in the course of the treatment of depression. Journal of Psychopharmacology, 13, 94-99.

Hennessy, S. \& Strom, B. (2007) PDUFA reauthorization - drug safety's golden moment of opportunity? New England Journal of Medicine, 356, 1703-1704.

Hughes, C., Emslie, G., Crismon, L., et al (2007) Texas children's medication algorithm project: update from Texas consensus conference panel on medication treatment of childhood major 
depressive disorder. Journal of the American Academy Child and Adolescent Psychiatry, 46, 667-686.

Institute of Medicine of the National Academies (2006) The Future of Drug Safety: Promoting and Protecting the Health of the Public. National Academies Press.

James, A. (2005) The use of selective serotonin re-uptake inhibitors (SSRIs) in the treatment of depressive disorders in children and adolescents. Epidemiologia e Psichiatria Sociale, 14, 63-66.

Jensen, P., Arnold, L., Swanson, J., et al (2007a) 3-year follow-up of the NIMH MTA study. Journal of the American Academy Child and Adolescent Psychiatry, 46, 989-1002.

Jensen, P., Youngstrom, E., Steiner, H., et al (2007b) Consensus report on impulsive aggression as a symptom across diagnostic categories in child psychiatry: implications for medication studies. Journal of the American Academy Child and Adolescent Psychiatry, 46, 309-322.

Keech, A., Gebski, V. \& Pike, R. (eds) (2007) Interpreting and Reporting Clinical Trials. A Guide to the CONSORT Statement and the Principles of Randomised Controlled Trials. MJA Books.

Kent, A. \& Mintes, B. (2007) Should patient groups accept money from drug companies? BMJ, 334, 934-935.

Laine, C. \& Horton, R. (2007) Clinical trial registration. BMJ, 334, 1177-1178

Libby, A., Brent, D., Morrato, E., et al (2007) Decline in treatment of pediatric depression after FDA advisory on risk of suicidality with SSRIs. American Journal of Psychiatry, 164, 884-891.

March, J. S., Silva, S. G., Compton, S., et al (2004a) The Child and Adolescent Psychiatry Trials Network (CAPTN). Journal of the American Academy Child and Adolescent Psychiatry, 43, 515-518.

March, J., Silva, S., Petrycki, S., et al (2004b) Fluoxetine, cognitivebehavioral therapy, and their combination for adolescents with depression: Treatment for Adolescents with Depression Study (TADS) randomized controlled trial. JAMA, 292, 807-820.

March, J., Kratochvil C., Clarke, G., et al (2004c) AACAP 2002 research forum: placebo and alternatives to placebo in randomized controlled trials in pediatric psychopharmacology. Journal of the American Academy Child and Adolescent Psychiatry, 43, 1046-1056.

March, J. S., Silva, S. G., Compton, S., et al (2005) The case for practical clinical trials in psychiatry. American Journal of Psychiatry, 162, 836-846.

March, J., Silva, S., Vitiello, B., et al (2006) The Treatment for Adolescents with Depression Study (TADS): methods and message at 12 weeks. Journal of the American Academy Child and Adolescent Psychiatry, 45, 1393-1403.

Majewicz-Hefley, A. \& Carlson, J. (2007) A meta-analysis of combined treatments for children diagnosed with ADHD. Journal of Attention Disorders, 10, 239-250.

McCracken, J., McGough, J., Shah, B., et al (2002) Risperidone in children with autism and serious behavioral problems. New England Journal of Medicine, 347, 314-321.

Morgan, S. \& Taylor, E. (2007) Antipsychotic drugs in children with autism. BMJ, 334, 1069-1070.
MTA Cooperative Group (1999) A 14-month randomized clinical trial of treatment strategies for attention-deficit/hyperactivity disorder. Multimodal Treatment Study of Children with ADHD. Archives of General Psychiatry, 56, 1073-1086.

MTA Co-operative Group (2004) National Institute of Mental Health Multimodal Treatment Study of ADHD follow-up: 24-month outcomes of treatment strategies for attentiondeficit/hyperactivity disorder. Pediatrics, 113, 754-761.

National Collaborating Centre for Mental Health (2004) Eating Disorders: Core Interventions in the Treatment and Management of Anorexia Nervosa, Bulimia Nervosa and Related Eating Disorders. British Psychological Society.

National Collaborating Centre for Mental Health (2005) Depression in Children and Young People: Identification and Management in Primary, Community and Secondary Care. British Psychological Society.

Olfson, M., Shaffer, D., Marcus, S., et al (2003) Relationship between antidepressant medication treatment and suicide in adolescents. Archives of General Psychiatry, 60, 978-982.

Olfson, M., Blanco, C., Liu, L., et al (2006) National trends in the outpatient treatment of children and adolescents with antipsychotic drugs. Archives of General Psychiatry, 63, 679-685.

Padina G., Aman, M. \& Findling R. (2006) Risperidone in the management of disruptive behavior disorders. Journal of Child and Adolescent Psychopharmacology, 16, 379-392.

Panorama (2007) 'Secrets of the drug trials'. BBC. http:/ / news. bbc.co.uk/1/hi/programmes/panorama/6291773.stm

Pediatric OCD Treatment Study Team (2004) Cognitive-behavior therapy sertraline, and their combination for children and adolescents with obsessive-compulsive disorder: the Pediatric OCD Treatment Study (POTS) randomized controlled trial. JAMA, 292, 1969-1976.

Posner, K., Oquendo, M., Gould, M., et al (2007) Columbia Classification Algorithm of Suicide Assessment (C-CASA): classification of suicidal events in the FDA's pediatric suicidal risk analysis of antidepressants. American Journal of Psychiatry, 164, 1035-1043.

Research Unit on Pediatric Psychopharmacology Anxiety Study Group (2001) Fluvoxamine for the treatment of anxiety disorders in children and adolescents. New England Journal of Medicine, 344, 1279-1285.

Shea, S., Turgay, A., Carroll, A., et al (2004) Risperidone in the treatment of disruptive behavioral symptoms in children with autistic and other pervasive developmental disorders. Pediatrics, 114, 1447-1448.

Timimi, S. (2007) Child psychiatry and its relationship with the pharmaceutical industry: theoretical and practical issues. Advances in Psychiatric Treatment, 14, 3-9.

Vieta, E. (2007) Psychiatry: from interest in conflict to conflicts of interest. World Psychiatry, 6, 27-29.

Wolpert, M., Fuggle, P., Cottrell, D., et al (2006) Drawing on the Evidence: Advice for Mental Health Professionals Working with Children and Adolescents (2nd edn.) CAMHS Publications. 